\title{
The Ontology of Counter Factual Causality and Conditional
}

\author{
Maduabuchi Dukor \\ Nnamdi Azikiwe University
}

\begin{abstract}
The author is interested here in the examination of the ontological justification for counterfactual causality and conditional. Ontology metaphysically has to do with what exists and what does not exist. In terms of realism, it is about objects in their associated and individual existence. It is an attempt to further the search for adequate philosophy of causality scientific law, and theory of confirmation and establish a link among if-then ">” and counterfactual.
\end{abstract}

Keywords: conditional, causality, counterfactual, ontology, science, explanation

\section{Introduction}

In theories concerning "cause and effect," a network of problems is encountered which I consider to be ontological problems of conditions, causality, and counterfactual. The different point of views about the meaning and truth value of a counterfactual presupposes an ontological problem. For instances, some believe that "counterfactuals are not truth-functional. A truth functional compound is one whose truth values are completely determined in every possible case by the truth value of its components" (Robert Audi 1995). On the other hand, Nelson Goodman opined that considered as truth—functional compounds, "all counterfactuals are of course true, since their antecedents are false” (1988), e.g., if that piece of butter had been heated to $150^{\circ} \mathrm{F}$, it would not have melted. The problems of counterfactuals are equally a problem of factual conditionals, for any counterfactual can be transposed into a conditional with a true antecedent and consequent, e.g., since that butter does not melt, it is not heated to $150^{\circ} \mathrm{F}$. The ontological question concerns the nature of the general statements that enable us to infer the consequent upon the basis of the antecedent and the statement of relevant conditions. The distinction between connecting principles and relevant conditions is imprecise and arbitrary because of its ontological nature. The pillars of science like causality and explanation are also appreciated more as ontological concepts when viewed from their relationship with conditional and counterfactual conditionals.

\section{Causality and Explanation}

There is an ontological connection between causality and explanation and what we call truth functional concept of causation is an explanation. According to Deshpands, causality is a natural relation that obtains between two events, things, state of affairs which are extra linguistic, natural, and extensional. Explanation on the other hand is non-natural, linguistics, intentional relations that hold among facts, truths, and prepositions. I think that a truth-functional logic will play a mediating role between causality and explanation. Logic explains causality with facts, truths, and propositions about the state of affairs and events. The definition of the concept

Maduabuchi Dukor, professor, Department of Philosophy, Nnamdi Azikiwe University, Nigeria; main research field: African Philosophy and Logic. Email: madudukor@yahoo.com. 
of causation with truth-functional logic presupposes the relationship between understanding and explanation. Causation is as much natural relation as relations of temporal succession or spatial proximity, e.g., that $t^{1}$ is to the left of $\mathrm{t}^{2}$ is a spatial relation and $\mathrm{c}^{1}$ before or after $\mathrm{c}^{2}$ is a temporal relation. In understanding causal phenomena as it is, we do explanations with facts, truth, and propositions about what we believe are natural relations and connections. The pertinent thing further is the modus operandi of explanation. What explains, i.e., explanans and what stands explained, i.e., explanandum are always truths, propositions, and facts. For instance, $\mathrm{c}$ and $\mathrm{e}$ are events in nature and there is a causal relation between them. Now when one gives an explanation as to why e, it is not e (an event) that is explained, but what is explained is the fact that e occurs at $t^{2}$. Similarly, when someone asks for an explanation for an event c, it is not c that stands explained but what is explained is the fact that occurs at $\mathrm{t}^{2}$. Hence, the relationship that obtains between two events is that of causation but not that of explanation. Yet it is believed that the borderline between explanation and causation is not clear.

The account of the difference among event, state of affairs and object on the other hand, and facts, truth, and proposition on the other hand is inadequate without an account of their common relationships to causation and explanation. I believe that facts truth and propositions of logic represent events, state of affairs and objects. Indeed, it has been argued by Lemmon that modern logic is characteristically concerned with arguments in mathematics and natural sciences, where there is a good reason to suppose that sentences do not wait upon the context of utterance for their truth values. Natural events and state of affairs and objects are expressible in language and propositions. David Hume says that the unique feature of causality as a natural relation is the idea of necessary connection of facts and truths. The language game concerning causality in Hume philosophy has synonyms like: efficiency, agency, power, force, energy, necessity, and connection. It has been argued that when the term "casual connection" is used, any one of four distinct objective relations may actually be meant, namely, “objectively, sufficient to, necessary to, necessitated by contingent upon” (Ducasse 1980).

These four relations correspond respectively to the four functional terms, cause, condition, effect, and resultant. The point remains that the four notions are notions of cause, but most importantly they can be reduced to two namely, "objectively sufficient" and "necessary to" where the former is a biconditional concept of causation and the later is a conditional concept, with "necessitated by" as a cause or antecedent of the biconditional "三” and "contingent upon" as the effect or the consequent of conditional (>). The ontological issue in the notion of "objectively sufficient" concept of causation is that there may be other unknown factor facilitating the process while the idea of necessity in the notion of "necessary to" is a modal term that is ontological without which it is meaningless.

\section{Cause and Effect Relationship}

There are causative principles in counterfactual conditional, scientific, general, and abstract laws. Lewis argues that an event c causes an event e only when e depends counterfactually on c and not otherwise. For simplicity and aesthetic reasons, these principles could be reduced to modal symbols to show the progressive causative principles in the relationships among "if-then," ">," "三,” and counterfactual conditional. What the above principles tend to show is that "if-then," “>,” “三,” and counterfactual conditional are different phases of causal law, not only laws of logic. For instance, "If that match had been scratched, it would have lighted" is a counterfactual conditional transposable to ">” and " $\equiv$ ” as follows: (a) If the match scratched, ">," it will light; (b)The match will light if "三” it is scratched. For Strawson, the "if-then," statement is neither false nor true, but only announces an intention and that "if $p$ then q" entails " $p>q$," but not conversely. I am suspicious of this 
position because the foundation is weak. It seems to me that “>” may be a causal law, “三” a stronger causal law and counterfactual conditional the strongest, being a necessity. They are all both laws in logic and in physics or science.

The idea of cause and effect is an ontological one (or metaphysical), so also the "if-then" or implication ">” which I believe defines the concept of cause and effect. Taylor argues that "if we can now cite clear examples of causal connections wherein the conditions that constitute the effect are entirely contemporary neither occurring before the other, then it will have been proved that the difference between a cause and its effect cannot be a temporal one, but must consist of something else” (Richard Taylor, 39). It is argued that the notion of cause and the notion of effect move together, but the notion of one is not temporally followed by the notion of the other.

However, Jagat Pal argues that the notion of causation cannot be defined in terms of counterfactual dependence. For instance, considering this proposition, "if wishes were horses (they are not), then beggars would ride. But its component propositions have nothing to do with any occurrence of events" (Pal Jagat, 501). Hence, according to him, the truth of counterfactual conditional does not always entail the truth of the causal statement. Even if we assume that counterfactual dependence holds only among occurrences, the occurrences need not be the occurrence of causal events. They may be numerical occurrences. For example, change in the volume of gasses depends counterfactually on the change in the temperature, and yet their relation is not a relation of cause and effect. The Boyle Charles Law of Gas is not a causal law because "It does not assert that a change in the temperature is followed (or preceded) by some change in the volume or in the pressure, it only asserts that a change in temperature is in concurrent with charges in pressure or volume or in both. But the law nonetheless does satisfy the condition of counterfactual dependence” (Pal Jagat, 501).

Hence for Jagat Pal, for c to be the cause of e, it is not at all logically required that c must be a necessary condition for the occurrence of e, because to say that certain conditions are necessary and certain conditions are not is not to say that the former are causes while the later are not. The notion of causes as such does not essentially include in its meaning the notion of a necessary connection. What it includes in its meaning is the force of causing and the force of causing does not essentially include in its meaning the notion of a necessary connection. This is because a thing can be conceived to be having the property of the force of causing certain events without being necessarily connected with it.

I think that the ultimate explanation available for the relation between cause and effect is ontological connection. It is perhaps, for ontological reason that some argue that the "notion of causality be expurgated from the philosophy of science on the ground either that it is too heavily loaded metaphysically or that it is too precise logically to have a place in exact thinking” (Wright, 95). Again the idea of replacing "cause and effect" with "functional relationship and probabilistic correlations" is another way of offering an ontological explanation to cause and effect. An ontological analysis of cause and effect implies that causal relations have a symmetry that is there will be nothing like problems of asymmetry of cause and effect. For ontological reasons, heavy rain could be the cause of flooding and conversely, without flood there would have been no heavy rain. But the symmetrical interpretation I will give here is that "no flooding” means "no heavy rain" and "heavy rain" means "flooding" thereby removing the notion of cause and retaining the notion of ontological symmetry.

\section{Causality and Counterfactual}

Counterfactuals, according to Lacey may be interpreted as "entailing or presupposing that their 
antecedents are false,” e.g., if Hitler had invaded England, Germany would have run and if I were you, I would run. Counterfactual conditionals seem more close to ordinary conditional than to material implications. As abstract as they are, they seem meaningless to the verification theory (positivism) and the correspondence theory of truth. Counterfactuals are said to be important in connecting with laws of nature, phenomenalism and dispositional statements like "The glass is brittle," which seems to imply that had it been struck, it would have broken. Some have distinguished natural law from accidental generalization (all the coins in my pocket are silver) by saying laws entail counterfactuals because on some views laws of nature do not make, and cannot entail assertion about the world. Counterfactuals could be hypothetical or nomological. "We cannot, without circularity, explain natural laws in terms of counterfactuals, and explain counterfactuals in terms of natural laws" (Lacey 1976). According to Goodman, the truth-value of counterfactuals does not derive from the truth-value of its compounds, for since the antecedent and the consequent of every counterfactuals are both false, all counterfactuals will have the same true value by every truth functional criterion. Therefore, counterfactuals connection must be defined in some quite different way. Then, what are the truths-conditions of counterfactual and how are they determined by its components? It is to be argued that there may be necessary and sufficient conditions under which counterfactual coupling of antecedent and consequence is warranted. A counterfactual conditional may be true if and only if the antecedent conjoined with the relevant true statement about the antecedent circumstances leads by way of a true general principle to the consequent.

According to the metalinguistic approach, or the deductive-nomological model of explanation, a counterfactual is true when its antecedent conjoined with issues of nature and statement of background condition logically entails its consequent, e.g., if the switch have been flipped, the light would be on. It is true because the statement that the switch was flipped plus the laws of electricity and the statement describing the condition and arrangement of the circuiting entails that the light is on. The issue here is an abstract one. When one talks of the conditions under which the electricity lights, one may not be able to cite all of them. It is possible that a lot of sufficient, necessary, and possible conclusions lead to the consequent, i.e., the light been on. It has been argued by the possible world semantic (modal logic) that "a counterfactual is true when a consequent is true in the nearest possible world in which the antecedent is true" (Goodman, 164). Therefore, the counterfactual about the switch is true on this account provided a world in which the switch is flipped and the light is on. Bob Hale has argued that the problem of modal knowledge is as much a problem about possibility as about necessity. He argues that "what is physically necessary is what is required by the laws of physics, i.e., what logically must be so, if there is to be no violation of physical law and what is physically possible is what can be so, without violation of any physical law, i.e., what is logically consistent with physical law" (Bob Hale, 2). In the context of this, do we say that flipping the switch in and the light on is physically necessary or possible? If it is physically necessary, is it necessary conditionally on the truth of certain propositions or is it being unconditionally necessary? I think that when we talk of this sort of counterfactual; conditional, by "truth of certain propositions," we also talk of ontological causality and relations between the antecedent and consequent. When the modality involved possibilities, i.e., when the switch is flipped, it is possible that light is on, then the causation involved is weak hence: the existentially generalized weak counterfactual. Henry Hiz says that a contrary-to-fact conditional might be interpreted as a metalinguistic statement, telling us something about what can be inferred in a given system of statement, "it says that if something is accepted in this system to be true, then something else can be accepted in this system to true" (Chisholm, 151). An assertion of a contrary-to-fact conditional goes like this "if A were S, A would be P." According to Chisholm, "The point of 
asserting the counterfactual may be that calling attention to, emphasizing, or conveying, one or more of the premises which, taken with the antecedent, logically imply the consequent" (Chisholm, 151). He says that "counterfactual is similar to probability statements in that each type of statement is, in a certain sense, elliptical" (Chisholm, 153). Here the attention calling to other premises not immediately perceives and the probability nature of the exercise presupposed the ontological nature of counterfactual conditionals. This is buttressed by the fact that "the formal properties of the conditional function, together with all the facts, may not be sufficient for determining the truth value of counterfactual..." (Stalnaker, 165). This is also an ontological and epistemological problem because "many counterfactuals seem to be synthetic and contingent, statement about unrealized possibilities” (Stalnaker, 166). They are literal and ontological statements about non-actual situations.

\section{Conclusion}

The above discussion is to show that counterfactuals in general and in particular conditional are general abstract or ontological principles and laws which establish the principle of causality. The thesis is suspicious of the thesis that "if $\mathrm{p}$ then q" entails " $\mathrm{p}>\mathrm{q}$," but not conversely. I think that so far as both have a counterfactual dependence, there is some measure of symmetric. Hence, “if-then," “>,” “三,”and counterfactual conditional, are different phases of casual law, not only laws of logic, that is, “>” may be a causal law, “三” a stronger causal law and counterfactual conditional, the strongest, born out of necessity.

\section{Works Cited}

Audi, Robert. The Cambridge Dictionary of Philosophy. Cambridge: Cambridge University Press, 1995. 163.

Chisholm, R. M. “Law Statements and Counterfactual Inference.” Ernest Sosa. England: Oxford University Press, 1980. 151.

Deshande, Shard. “Causation, Explanation, and Understanding.” Indian Philosophical Quarterly, Vol. XXII, No. 4. 1995.

Ducasse, C. J. "On the Nature and the Absorbability of the Causal Relation.” Causation and Conditionals. Ernest Sosa, ed.. Oxford Readings in Philosophy. England: Oxford University Press, 1980. 115.

Goodman, Nelson. Fact, Fiction and Forecast. England: Harvard University Press, 1988. 4.

Hale, Bob. "Knowledge of Possibility and of Necessity.” Proceedings of the Aristotelian Society, Vol. III, Part 1. 2003.

Jagat Pal. “D. Lewise on Causeation.” Indian Philosophical Quarterly, Vol. XXVI No. 4, Oct, 1999. 495.

Lacey, A. R. A. Dictionary of Philosophy. London: Routledge and Regan Paul Ltd., 1976, 36.

Lemon. “Sentences, Statements and Proposition.” Readings in the Philosophy of Language. J. F. Rosenberg and C., 1971.

Robert Audi. The Cambridge Dictionary of Philosophy. England: Cambridge University Press, 1995. 163.

Stalnaker, R. C. “A Theory of conditional.” Ernest Sosa. New Jersey: Prentice Hall, Inc., 1980. 237.

Wright, G. H. Von. “On the Logic and Epistemology of the Causal Relation.” Ernest Sosa. England: Oxford University Press, 1980. 95. 